\title{
Patterns, Predictors and Outcome of Time to Presentation Among Critically ill Paediatric Patients at Emergency Department of Muhimbili National Hospital, Dar es Salaam, Tanzania
}

\section{Alphonce N Simbila}

Muhimbili University of Health and Allied Sciences

Said S. Kilindimo ( $\nabla$ skilindimo@yaho.com )

Muhimbili University of Health and Allied Sciences

Hendry R. Sawe

Muhimbili University of Health and Allied Sciences

Zawadi E. Kalezi

Muhimbili University of Health and Allied Sciences

Amne 0. Yussuf

Muhimbili University of Health and Allied Sciences

Hussein K. Manji

Muhimbili University of Health and Allied Sciences

Germana Leyna

Muhimbili University of Health and Allied Sciences

Juma A. Mfinanga

Muhimbili National Hospital

Ellen J. Weber

Muhimbili National Hospital

\section{Research Article}

Keywords: Paediatric patient, critically ill, delay, mortality

Posted Date: November 10th, 2021

DOI: https://doi.org/10.21203/rs.3.rs-1055542/v1

License: (1) This work is licensed under a Creative Commons Attribution 4.0 International License.

Read Full License 


\section{Abstract}

Background: Mortality among under-five children in Tanzania remains high. While early presentation for treatment increases likelihood of survival, delays to care are common and factors causing delay to presentation among critically ill children are unknown.

Methodology: This was a prospective cohort study of critically ill children aged 28days to 14 years attending emergency department (ED) at Muhimbili National Hospital in Tanzania from September 2019 to January 2020. We documented demographics, time to ED presentation, ED interventions and 30-day outcome. The primary outcome was delay ( $>48$ hours) from the onset of illness to ED presentation. Logistic regression and relative risk were calculated to measure the strength of the predictor and relationship between delay and mortality respectively.

Results: We enrolled 440 (59.1\%) critically ill children, their median age was 12 [IQR $=9-60$ ] months and $63.9 \%$ were males. The median time to ED arrival was 3 days [IQR $=1-5]$ and more than half $(56.6 \%)$ of critically ill children presented to ED in $>48$ hours where by being an infant, self-referral and belonging to poor family were independent predictors of delay. Infants and those referred from other facilities had 2.2 (95\% $\mathrm{Cl} 1.3-3.8)$ and 1.7 (95\% $\mathrm{Cl} 1.1-2.7)$ times increased odds of presenting late to the ED respectively. The overall 30-day in-hospital mortality was $26.5 \%$ in which those who presented late were 1.3 more likely to die than those who presented early $(\mathrm{RR}=1.3, \mathrm{Cl}: 0.9-1.9)$. Majority died $>24$ hours of ED arrival (P-value $=0.021)$.

Conclusion: Delayed ED presentation of more than 48 hours from onset of illness was associated with inhospital mortality. A larger study is needed to evaluate the care pathway of critically ill paediatric patients to identify preventable course of delay to tertiary care facility.

\section{Background}

Critical illness is a life-threatening process which, without timely medical or surgical intervention, is highly likely to result in death(1). Half of the deaths among children under five years globally occurred in subSaharan Africa in 2017. Unlike in high income countries where 1 in 185 children died before the age of five years, 1 in 13 died before the age of five in Sub-Saharan Africa. The risk of dying for a child in subSaharan Africa is 15 times higher than in Europe. Most of these children die due to treatable and preventable causes such as complications of during birth, pneumonia, diarrhea, malaria and neonatal sepsis(2). A 2010 survey by the ministry of health in Tanzania showed high mortality rate in under-fives with $75 \%$ of deaths occurring in the first 24 to 48 hours after admission(3). The unfolding of events in the process of critical illness is influenced by multiple factors which can potentially modify and affect the outcome of critical illness. In addition to improvements in the quality of care for children reducing delays in critical illness is among the main approaches to reducing mortality.

Paediatric critical illness in the LMICs differ from the developed countries in that children tend to be younger and suffer more from infectious causes of illnesses. Late presentations to the hospital due to 
referrals and travels through long distances to reach hospitals are common occurrence which contribute to an increased disease severity and mortality upon admission(4).

Delayed presentation to the hospital in the course of paediatric critical illness has been shown to be one of the factors that negatively influence health outcomes(5). Delayed health care seeking of more than 48 hours has been observed in $35 \%$ of paediatric patients in the acute phase of illness in Kigali, Rwanda(6). At a tertiary hospital in Ethiopia about 4. 1\% of children died at the Paediatric Emergency Department, which translates to a mortality of 8.2 per 1000 children. Delay of more than 48 hours since the onset of symptoms was among the top causes of early mortality(5). In Southwestern Uganda, $50 \%$ of paediatric patients who presented late to the health facility and were admitted died within 24 hours of admission(7). In Dodoma Tanzania, the median time of delay to seek care among children under five years with fever was 2 days(8).There is an association between delayed ICU admission and mortality. Fraction of mortality attributable to ICU delay was 30\%(9). Many studies in this area have looked at timeliness at the level of the family which affects the first phase in the Thaddeus and Maine's delay model(10).

Presence of a full capacity ED, Paediatric ICU and definitive paediatric services at MNH have provided early resuscitation, stabilization and other management for critically ill paediatric patients. The objective of this study was to describe patterns, determine factors associated with delay and find out whether delay made a difference in mortality outcome among critically ill children who sought care at the ED of a national tertiary referral hospital.

\section{Methods}

\section{Study design}

This was a prospective observational cohort study of paediatric patients aged 28days to 14 years presented to the MNH ED from September 2019 to January 2020 and triaged level 1 according to Emergency Severity Index (ESI).

\section{Study setting}

The study was conducted at ED of MNH which is a national tertiary referral government hospital located in Dar es Salaam with 1500 bed capacity. The MNH serves an annual average of 60,000 patients who are referred from all over the country. The ED at MNH is full capacity public ED in Tanzania and the only training site for Emergency Medicine residency program in the country. On a daily basis the MNH ED attends about $150-200$ critically ill patients, among them approximately $25 \%$ are children excluding neonates who present directly to the maternity unit. The MNH ED uses a triage system modelled on the ESI but with only 3 levels, with emergent being equivalent to levels 1 and 2 of ESI, and they are assigned to the resuscitation room. Apart from ability to provide full resuscitation including continuous cardiopulmonary monitoring, the department also has a full range of point of care tests like ultrasound, chemistry, blood gas analyzer and portable x-ray. After resuscitation and stabilization, the critically ill children get admitted to general pediatrics ward or pediatric intensive care unit (PICU). 


\section{Study Participants}

We included all pediatric patients (aged 28 days to 14 years) triaged as emergent whose parents/guardians consented to participate in the study.

\section{Study protocol}

Consecutive sampling technique was employed to enroll patient $s$ where by data collected for 24 hours/day on alternate days. Demographics, clinical presentation, initial management, and ED outcomes were observed and documented using information given by the parent/guardian, the treating physician, and the electronic medical record (Wellsoft ${ }^{\mathrm{TM}}$ ) using structured case report form. All patients were asynchronously followed up in hospital wards (if admitted) to determine their in-hospital outcome (discharge/mortality) in 24 hours and later weekly for a maximum period of 1 month after admission.

\section{Measurements:}

Each caretaker of a critically ill paediatric patient was asked about the date and time of onset of an index illness of the child. Time of presentation to the MNH ED was therefore calculated by finding the difference between these. Delay was defined as presentation to MNH ED after 48 hours from onset of illness. Wealth was determined based on household characteristics and asset ownership hence relative household wealth index was constructed using principal component analysis (PCA). Households were then ranked in ascending order. The scores were separated into quintiles; each representing $20 \%$ of the population. Those in the highest quintile might not have been rich but were in higher socioeconomic status than $80 \%$ of the participants in this study.

\section{Outcomes}

The primary outcome was the association of delay with mortality and secondary outcomes was predictors of delay among critically ill paediatric patients.

\section{Data analysis}

Data were imported into the Statistical Package for Social Science for analysis (SPSS) (version 26.0, IBM, LTD, North Carolina, USA) from the Research Electronic Data Capture (RedCap version 7.2.2, Vanderbilt, Nashville, TN, USA). Relevant frequencies and tables were generated for categorical variables (injury and referral factors). Medians/inter-quartile ranges were calculated for continuous variables. We calculated a proportion of children with delayed presentation and contingency tables were constructed for univariate analysis to explore differences between children who had a delayed vs. timely presentation using the Chisquare test. Multivariate logistic regression analysis was completed on variables with $p$ value $\leq 0.20$ in the univariate analysis to identify independent predictors of delayed presentation. Relative risks were computed for association of delay with overall, early and late mortality. The odd ratios and $95 \%$ confidence intervals were estimated for each studied factor. Statistical significance was set at $\mathrm{p}$ value $<0.05$. 


\section{Results}

A total of 3616 paediatric patients attended the ED during the study period, of whom 745 (20.6\%) were triaged "emergent". A total of $440(59.1 \%)$ patients were eligible and consented to participate in the study. Of the $99(26.5 \%)$ who died within 30 days of presentation, $64(29.5 \%)$ presented late (after 48 hours) and $35(22.3 \%)$ presented early (before 48 hours). (Figure 1 )

\section{Socio-demographic characteristics of critically ill paediatric patients at the MNH ED and their caretakers}

In the studied cohort, majority 281(63.9\%) were males, the median age was 12 [IQR $=9-60]$ months and majority $321(73.0 \%)$ were referred from a lower-level health facility. Most of the caretakers of the critically ill paediatric patients were parents $410(93.2 \%)$ and more than half of them $260(59.1 \%)$ were between the age of 25 and 34 years. Slightly more than half of them $236(53.6 \%)$ had primary education, $145(33.0 \%)$ were unemployed, 46(10.5\%) were peasants and 59 (67.8\%) were the poorest in the socioeconomic status. (Table 1) Of the paediatric patients, $72 \%$ were admitted to the ward, $19 \%$ were admitted to the PICU and 9\% (33/374) died while receiving care at the ED. 
Table 1

Socio-demographic characteristics of paediatric patients with ESI triage level 1 at MNH ED

\begin{tabular}{|c|c|c|c|}
\hline \multirow[t]{2}{*}{ Variable } & \multirow[t]{2}{*}{ Category } & \multirow[t]{2}{*}{ Median [IQR] } & \multirow{2}{*}{$\begin{array}{l}\text { Frequency (\%) } \\
\mathrm{N}=440\end{array}$} \\
\hline & & & \\
\hline \multirow[t]{4}{*}{ Age (months) } & $<12$ & & $142(32.3)$ \\
\hline & $12-<0$ & & $181(41.1)$ \\
\hline & $\geq 60$ & & $117(26.6)$ \\
\hline & Median [IQR] & $12(9-60)$ & \\
\hline \multirow[t]{2}{*}{ Sex } & Male & & $281(63.9)$ \\
\hline & Female & & $159(36.1)$ \\
\hline \multirow[t]{2}{*}{ Type of referral } & Facility & & $321(73.0)$ \\
\hline & Self-referral & & $119(27.0)$ \\
\hline \multirow[t]{2}{*}{ Caretaker } & Parent & & $410(93.2)$ \\
\hline & Guardian & & $30(6.8)$ \\
\hline \multirow[t]{4}{*}{ Age of caretaker (years) } & $<25$ & & $47(10.7)$ \\
\hline & $25-34$ & & $260(59.1)$ \\
\hline & $\geq 35$ & & $133(30.2)$ \\
\hline & Median [IQR] & $32(28-36.7)$ & \\
\hline \multirow[t]{4}{*}{ Level of education of caretaker } & No formal education & & $32(7.3)$ \\
\hline & Primary education & & $236(53.6)$ \\
\hline & Secondary education & & $141(32.0)$ \\
\hline & University/college & & $31(7.0)$ \\
\hline \multirow[t]{4}{*}{ Occupation status of caretaker } & Employed & & $55(12.5)$ \\
\hline & Self employed & & $194(44.1)$ \\
\hline & Unemployed & & $145(33.0)$ \\
\hline & Peasant & & $46(10.5)$ \\
\hline \multirow[t]{4}{*}{ Socioeconomic status } & Poorest & & $87(19.8)$ \\
\hline & Poor & & $91(20.7)$ \\
\hline & Medium & & $92(20.9)$ \\
\hline & Rich & & $79(18.0)$ \\
\hline
\end{tabular}




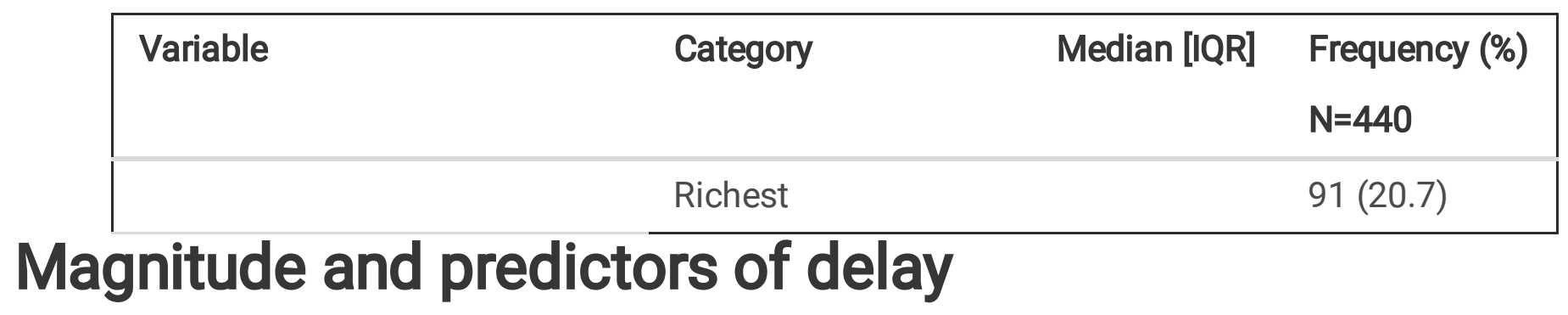

Among the 440 critically ill children, 249/440 (56.6\%) had delayed presentation with median time interval from onset of illness until presenting to the ED - MNH was 3 days with IQR [1-5]. After multivariate logistic regression, aless 1 year old, referred patients, and poor socioeconomic status were independent predictors of delay presentation with OR 2.2, 1.8 and 2.24 respectively. (Table 2 and 3 ) 
Table 2

Predictors of delayed presentation to MNH ED among paediatric patients with ESI triage level 1

\begin{tabular}{|c|c|c|c|c|}
\hline \multirow[t]{2}{*}{ Variable } & \multicolumn{2}{|c|}{ Timeliness, $\mathbf{N}(\%)$} & \multirow[t]{2}{*}{ OR $(95 \% \mathrm{Cl})$} & \multirow[t]{2}{*}{ p-value } \\
\hline & Early & Late & & \\
\hline Age of a child (Months) & $\mathrm{N}=191$ & $\mathrm{~N}=\mathbf{2 4 9}$ & & \\
\hline$<12$ & $48(33.8)$ & $94(66.2)$ & $2.2(1.3-3.7)$ & 0.002 \\
\hline $12-60$ & $81(44.8)$ & $100(55.2)$ & $1.4(0.9-2.2)$ & 0.17 \\
\hline$\geq 60 *$ & $62(53.0)$ & $55(47.0)$ & & \\
\hline \multicolumn{5}{|l|}{ Sex } \\
\hline Male & $131(46.6)$ & $150(53.4)$ & $0.7(0.5-1.0)$ & 0.07 \\
\hline Female* & $60(37.5)$ & $99(62.3)$ & & \\
\hline \multicolumn{5}{|l|}{ Type of referral } \\
\hline Self-referral* & $66(55.5)$ & $53(44.5)$ & & \\
\hline Facility referral & 125(38.9) & 196(61.1) & $2.0(1.3-3.0)$ & 0.002 \\
\hline \multicolumn{5}{|l|}{ Level of education of a caretaker } \\
\hline No formal education* & $10(31.3)$ & $22(68.8)$ & & \\
\hline Primary education & $101(42.8)$ & $135(57.2)$ & $0.6(0.3-1.3)$ & 0.20 \\
\hline Secondary and higher education & $80(46.5)$ & $92(53.5)$ & $0.5(0.2-1.2)$ & 0.11 \\
\hline \multicolumn{5}{|l|}{ Occupation status of a caretaker } \\
\hline Employed & $30(54.5)$ & $25(45.5)$ & $0.7(0.4-1.2)$ & 0.16 \\
\hline Self-employed/ Business* & $85(43.8)$ & $109(56.2)$ & & \\
\hline Unemployed & $76(39.8)$ & $115(60.2)$ & $1.2(0.8-1.8)$ & 0.42 \\
\hline \multicolumn{5}{|l|}{ Socioeconomic status } \\
\hline Poorest & $28(32.2)$ & $59(67.8)$ & $2.4(1.3-4.3)$ & 0.006 \\
\hline Poor & $40(44.0)$ & $51(56.0)$ & $1.4(0.8-2.6)$ & 0.24 \\
\hline Medium & $41(44.6)$ & $51(55.4)$ & $1.4(0.8-2.5)$ & 0.27 \\
\hline Rich & $34(43.0)$ & $45(57.0)$ & $1.5(0.8-2.7)$ & 0.21 \\
\hline Richest* & $48(52.7)$ & $43(47.3)$ & & \\
\hline
\end{tabular}


Table 3

Multivariate analysis of predictors of delayed presentation to MNH ED among paediatric

\begin{tabular}{|ll|}
\hline Variable & OR $(95 \% \mathrm{Cl})$ \\
\hline Age (Months) & $2.4(1.4-4.0)$ \\
\hline$<12$ & $1.5(0.9-2.5)$ \\
\hline $12-60$ & $0.7(0.5-1.0)$ \\
\hline Sex (Male) & $1.8(1.1-2.8)$ \\
\hline Facility referral & \\
\hline Level of education of a caretaker & $0.7(0.3-1.6)$ \\
\hline Primary education & $0.9(0.3-2.2)$ \\
\hline Secondary and higher education & \\
\hline Occupation status of a caretaker & \\
\hline Employed & $1.0(0.7-1.6)$ \\
\hline Unemployed & $0.7(0.4-1.4)$ \\
\hline Socioeconomic status & \\
\hline Poorest & $2.4(1.2-4.8)$ \\
\hline Poor & $1.4(0.8-2.7)$ \\
\hline Medium & $1.7(0.9-3.1)$ \\
\hline Rich & $1.5(0.8-2.8)$ \\
\hline
\end{tabular}

Table 4

Association of delay with overall mortality among paediatric patients with ESI triage level 1

\begin{tabular}{|llll|}
\hline Variable & \multicolumn{2}{c}{ Mortality N (\%) } & Relative Risk (95\% Cl) \\
\cline { 2 - 3 } & Dead $(\mathrm{N}=99)$ & Alive $(\mathrm{N}=275)$ & \\
\cline { 1 - 3 } Delayed presentation & $64(29.5)$ & $153(70.5)$ & \multirow{2}{*}{$1.3(0.9-1.9)$} \\
\cline { 1 - 3 } Early presentation & $35(22.3)$ & $122(77.7)$ & \\
\hline
\end{tabular}

\section{Mortality and delay among critically ill paediatric patients}

Of all critically ill patient, 374 (85\%) completed follow up, among them 44(11.8\%) died within 24 hours which makes total of $99(26.5 \%)$ died within 30 days. Of those who died, $64(64.6 \%)$ presented late to the 
ED which have 1.5 times more likely to die compared to those who came early. (RR 1.5 (95\% Cl: 0.9-2.4) (Table 3).

\section{Discussion}

Delay to seek care has been reported in several studies as a cause of avoidable morbidity and mortality in children and many of these studies was done in higher income countries with limited information in lowand middle-income countries. In our study over fifty percent of critically ill paediatric patients presented late (after 48 hours from onset of illness) to the ED. This is similar to findings by a study in Ethiopia but higher than that observed in Rwanda with delay of thirty five percent of paediatric patients. $(5,6)$. A possible explanation could be differences in sociodemographic characteristics and lifestyle of the study settings. The hierarchical referral system in the setting of this study could be contribute to the observed delay as this study was conducted in tertiary hospital which is the highest destination in the chain of referral(11).

In this cohort almost a quarter of the critically ill children who presented to the ED after 48 hours from onset of illness died with almost two- thirds of the deaths occurred beyond 24 hours of hospital admission. This is similar findings to the study done at a tertiary hospital in Ethiopia(5). This may signify the impact of resuscitation and stabilization at the ED before admission that presumably prolonged their lives beyond 24 hours but later decompensated(13). However, limited number of beds in paediatric ICU could explain the occurrence of death beyond ED stabilization

We also found that critically ill children below one year of age were more likely to present late to the ED. Non-specific symptoms especially in infants and caretakers' poor knowledge on danger signs of critical illnesses might contribute to delay in this age group (14). Our study also found coming from the poorest households independently doubled the odds of a critically ill paediatric patient being late to the tertiary hospital during a critical illness. This is in keeping with a study conducted in Ethiopia which had similar findings(15).

About two thirds of the critically ill paediatric patients who delayed were referred from primary health care facilities with no capability to care for critically ill children. Failure to recognise critical illness with series of evaluations by primary health care providers before they are referred to definitive care significantly lead to delay with limited management (16). The existing referral system in place (11) doesn't take into account the urgency to definitive care hence more delay with poor outcome observe.

\section{LIMITATIONS}

This study was conducted in urban settings and single center tertiary hospital with full capacity to resuscitate and stabilize critically ill paediatric patients. However, the MNH ED receives referrals from all over the country, the patients sampled are likely to provide a true representation of the Tanzanian population of critically ill paediatric patients. 
Patients were only enrolled when researchers were in the department; some of potential participants might have been missed. However, as the researchers worked different shifts, there is no reason to expect that the missed patients were different from those that were enrolled.

There was loss to follow up encountered in this study. However, it was mitigated by including $10 \%$ loss to follow up rate during the estimation of the sample size of the study participants who were to be included during the development of the study protocol.

\section{Conclusion}

The proportion of delayed presentation to tertiary hospital among critically ill paediatric patients is substantially high. Age below one year, being referred from a primary healthcare facility and being in the poorest category of socioeconomic status predicted delay to appropriate care facility. Future studies should focus on the evaluation of care pathway among critically ill paediatric patients to identify preventable delays in care.

\section{Declarations}

\section{Competing interests}

The author declares no conflicts of interest

\section{Ethics approval and consent to participate}

The study was conducted after obtaining permision from the MUHAS Institutional Review Board and $\mathrm{MNH}$. All patients were entered into the study after an informed consent was signed either by the patients themselves or their guardians in the case where patients were not able to do so. The investigator received the waiver of consent from the MUHAS Institutional Review Board incase patients weren't able to consent such as unconscious patients with absence of relatives to provide consent. Only participants who on their free will consent were included in the study. Confidendiality was observed on the questionnaires filled with participants information. All patients received treatment as per standard hospital policies. The data obtained during the study was kept anonymous.

\section{Consent to publish:}

Not applicable

\section{Availability of data and material}

The dataset supporting the conclusion of this article is available from the authors on request 


\section{Funding}

This was a non-funded project; the principal investigators used their own funds to support the data collection and logistics

\section{Authors' contributions}

ANS was involved in the study design conceptualization, data collection, analysis and interpretation, drafted the manuscript, and made all necessary changes to the manuscript. SSK was involved in the study design conceptualization, review of the data analysis and interpretation, and critical review of the manuscript. HRS was involved in the study design conceptualization, review of the data analysis and interpretation, and critical review of the manuscript. JAM was involved in review of the data analysis and interpretation, and critical review of the manuscript. ZEK and GL were involved in the study design conceptualization, data analysis and interpretation and revision of the manuscript. AOY were involved in study design conceptualization and revision of manuscript. HKM were involved in study design conceptualization and revision of manuscript. EJW was involved in the study design conceptualization, review of the data analysis and interpretation together with revision of the manuscript. All authors have read and approved the final manuscript.

\section{Acknowledgements}

The author would like to thank all the study participants and research assistants for making this project a success.

\section{References}

1. Robertson LC, Al-haddad M. Recognizing the critically ill patient. Anaesth Intensive Care Med [Internet]. 2013;14(1):11-4. Available from: http://dx.doi.org/10.1016/j.mpaic.2012.11.010

2. UNICEF. Levels \& Trends in Child Mortality: Report 2019-Estimates developed by the UN Inter-agency Group for Child Mortality Estimation. Unicef/Who/Wb/Un. 2019;1-32.

3. Tanzania M of $\mathrm{H}$ and SW. WHO Baseline survey on quality of paediatric care in Tanzania. Minist Heal Soc Welfare, Dar es Salaam, United Repub Tanzania [Internet]. 2010;(November). Available from: http://www.who.int/childmedicines/countries/QUALITY_TANZANIA.pdf

4. Royal C, McKerrow NH. A retrospective review of the transfer of critically ill children to tertiary care in KwaZulu-Natal, South Africa. SAJCH South African J Child Heal. 2015;9(4):112-8.

5. Jofiro G, Jemal K, Beza L, Heye TB. Prevalence and associated factors of pediatric emergency mortality at Tikur Anbessa specialized tertiary hospital: a 5 year retrospective case review study. 2018;1-10. 
6. Umuhoza C, Karambizi AC, Tuyisenge L, Cartledge P. Caregiver delay in seeking healthcare during the acute phase of pediatric illness, Kigali, Rwanda. Pan Afr Med J. 2018;30:160.

7. Wiens MO, Gan H, Barigye C, Zhou G, Kumbakumba E, Kabakyenga J, et al. A cohort study of morbidity, mortality and health seeking behavior following rural health center visits by children under 12 in southwestern Uganda. PLoS One. 2015;10(1):1-15.

8. Kassile T, Lokina R, Mujinja P, Mmbando BP. Determinants of delay in care seeking among children under five with fever in Dodoma region, central Tanzania : a cross-sectional study. 2014;1-10.

9. Cardoso LTQ, Grion CMC, Matsuo T, Anami EHT, Kauss IAM, Seko L, et al. Impact of delayed admission to intensive care units on mortality of critically ill patients: A cohort study. Crit Care. 2011;15(1):1-8.

10. Thaddeus S, Maine D. Too To Walk: Maternal Mortality in. Soc Sci Med. 1994;38(8):1091-110.

11. A CLOSER LOOK AT THE HEALTHCARE SYSTEM IN TANZANIA. 2016;(October):2016.

12. Kutko MC, Calarco MP, Flaherty MB, Helmrich RF, Ushay HM, Pon S, et al. Mortality rates in pediatric septic shock with and without multiple organ system failure. 2003;4(3):333-7.

13. Date P. Western Journal of Emergency Medicine: Integrating Emergency Care with Population Health Impact of Post-Intubation Interventions on Mortality in Patients Boarding in the Emergency Department. 2014;

14. Wambui WM, Kimani S, Odhiambo E. Determinants of Health Seeking Behavior among Caregivers of Infants Admitted with Acute Childhood Illnesses at Kenyatta National Hospital, Nairobi, Kenya. 2018;2018.

15. Deressa W, Ali A, Berhane Y. Household and socioeconomic factors associated with childhood febrile illnesses and treatment seeking behaviour in an area of epidemic malaria in rural Ethiopia. 2007;

16. Hodkinson P, Argent A, Wallis L, Reid S, Perera R, Harrison S, et al. Pathways to care for critically III or injured children: A cohort study from first presentation to healthcare services through to admission to intensive care or death. PLoS One. 2016;11(1):1-16.

\section{Figures}




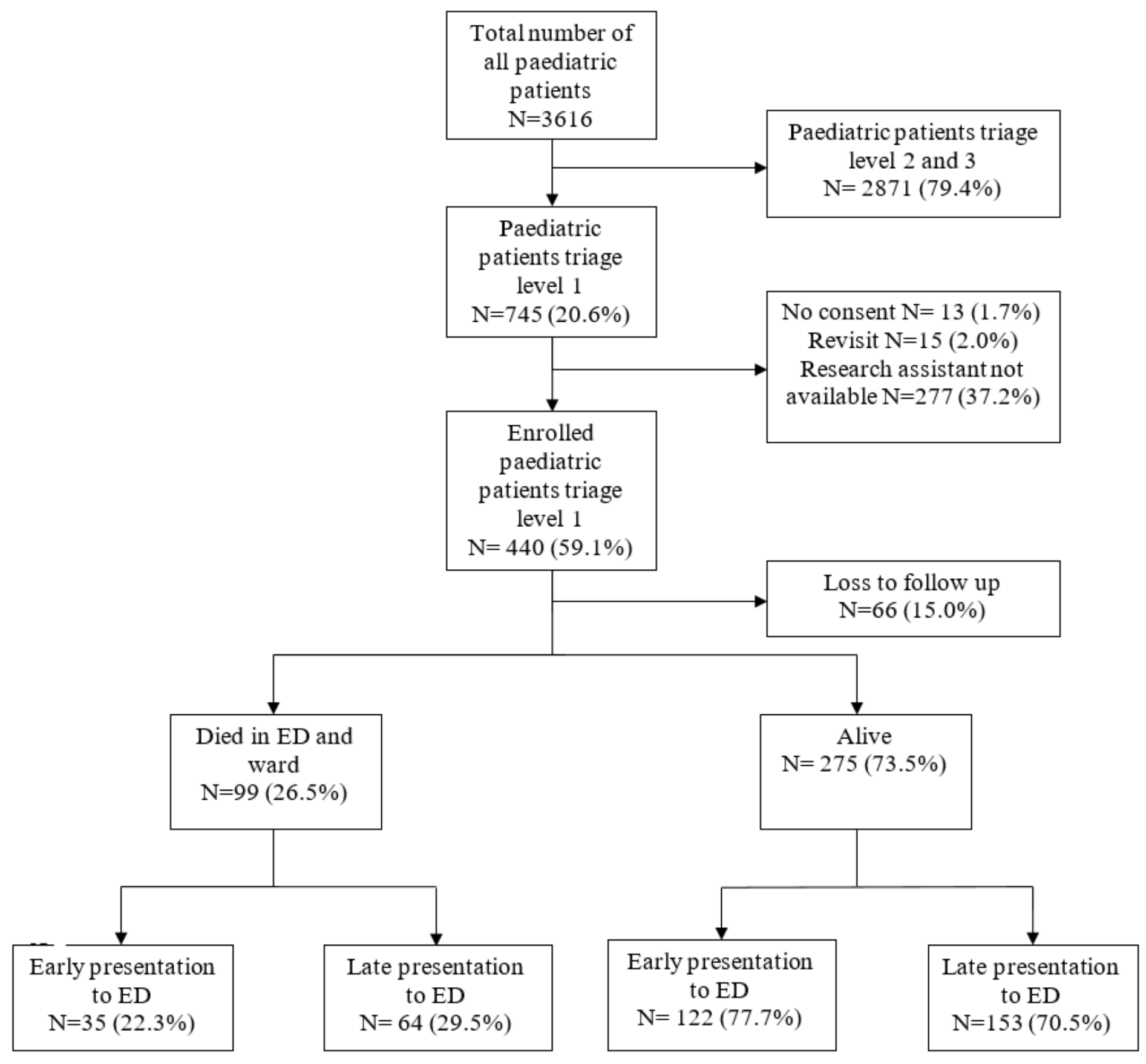

Figure 1

Flow chart of paediatric patients 


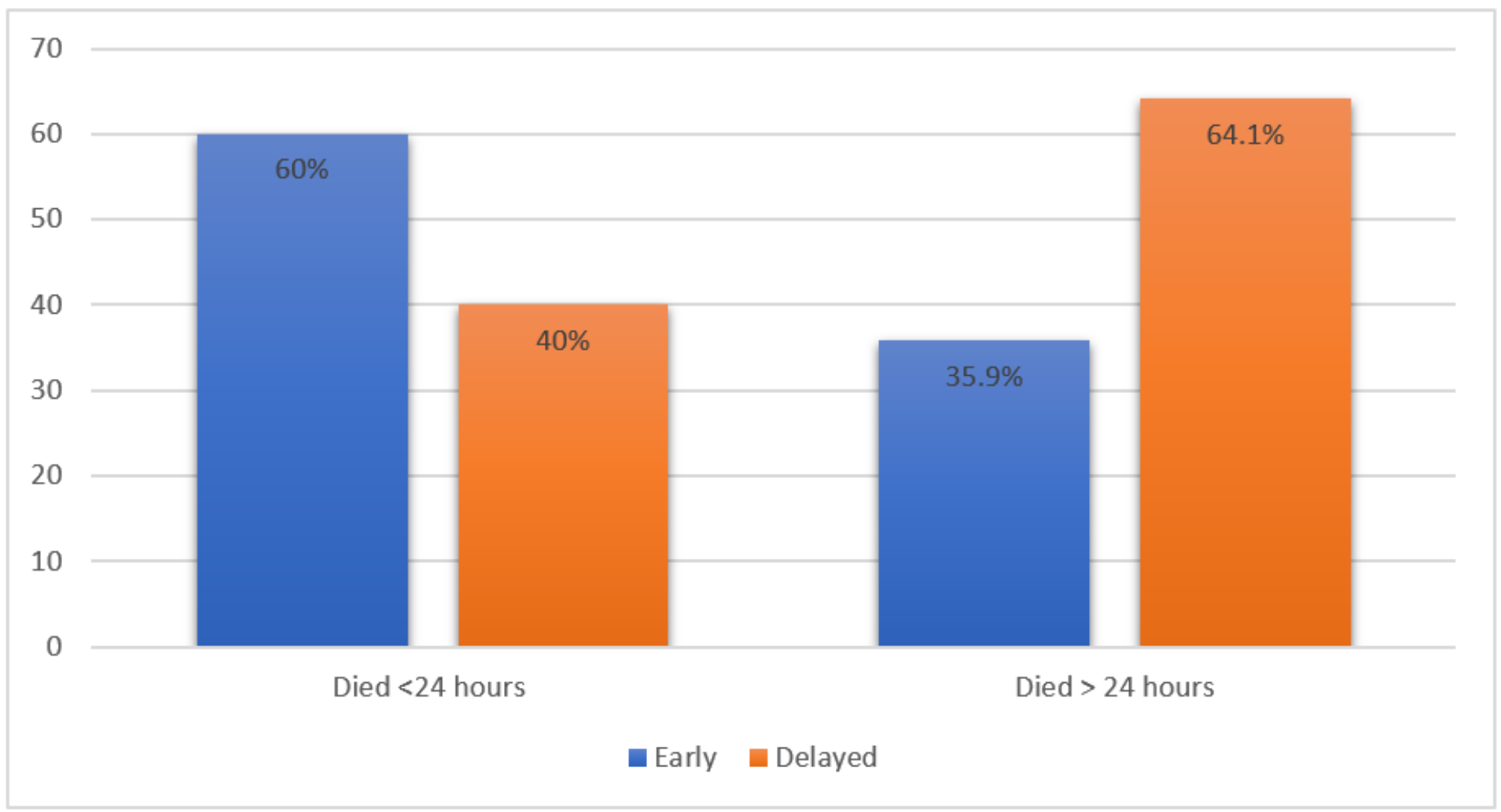

\section{Figure 2}

Distribution of early and late mortality vs early and late presentation among paediatric patients with ESI triage level $1 *(p-v a l u e=0.021)$ 